\title{
Positron-lifetime study of compensation defects in undoped semi-insulating InP
}

\author{
C. D. Beling, A. H. Deng, Y. Y. Shan, Y. W. Zhao, and S. Fung \\ Department of Physics, The University of Hong Kong, Hong Kong, China \\ N. F. Sun, T. N. Sun, and X. D. Chen \\ Hebei Semiconductor Research Institute, P.O. Box 179, ShijiaZhaung, People's Republic of China
}

(Received 5 June 1998)

\begin{abstract}
Positron-lifetime and infrared-absorption spectroscopies have been used to investigate the compensation defects that render undoped $n$-type liquid encapsulated Czochralski-grown InP semi-insulating under hightemperature annealing. The positron measurements, carried out over the temperature range of $25-300 \mathrm{~K}$, reveal in the as-grown material a positron lifetime of $282 \pm 5$ ps which we associate with either the isolated indium vacancy $V_{\mathrm{In}}^{3-}$ or related hydrogen complexes. The shallow donor complex $V_{\mathrm{In}} \mathrm{H}_{4}$, responsible for much of the $n$-type conductivity and the strong infrared absorption signal at $4320 \mathrm{~nm}$, is ruled out as a significant trapping site on the grounds that its neutral state is present at too low a concentration. After annealing at $950{ }^{\circ} \mathrm{C}$, in conjunction with the disappearance of the $V_{\text {In }} \mathrm{H}_{4}$ infrared-absorption signal, trapping into $V_{\text {In }}$-related centers is observed to increase slightly, and an additional positron trapping defect having a lifetime of 330 ps appears at a concentration of $\sim 10^{16} \mathrm{~cm}^{-3}$, indicating divacancy trapping. These results support the recent suggestion that the $V_{\mathrm{In}} \mathrm{H}_{4}$ complex present in as-grown InP dissociates during annealing, forming $V_{\mathrm{In}} \mathrm{H}_{n}^{(3-n)-}(0 \leqslant n \leqslant 3)$ complexes and that the recombination of $V_{\text {In }}$ with a phosphorus atom results in the formation of EL2-like deep donor $\mathrm{P}_{\mathrm{In}}$ antisite defect, which compensates the material. It is suggested that the divacancy formed on annealing is $V_{\mathrm{In}} V_{\mathrm{P}}$, and that this defect is probably a by-product of the $\mathrm{P}_{\mathrm{In}}$ antisite formation.
\end{abstract}

[S0163-1829(98)02044-X]

\section{INTRODUCTION}

In recent years, InP has been considered a potentially important material for the development of optoelectronic devices. Such devices often require InP in semi-insulating (SI) form as a substrate material. Until recently the only method of forming SI-InP has been through Fe doping, in which deep acceptors compensate for the residual free electrons that are present even in the purest undoped as-grown substrates. However, the presence of the Fe dopant often affects device performance. $^{1,2}$ The discovery that a semi-insulating state of the undoped liquid encapsulated Czochralski (LEC)-grown InP can be achieved by annealing at $800-900{ }^{\circ} \mathrm{C}$ in vacuum or phosphorus ambient ${ }^{3-7}$ is thus a very significant breakthrough in InP technology. Although a great deal of interest has been attracted by this transition from $n$-type to semiinsulating conductivity, the role of defects involved in the compensation mechanism is still poorly understood and under investigation.

As a sensitive probe for vacancylike defects, the positronannihilation technique can be employed to obtain microscopic information of defects, such as vacancy size, concentration, charge states, and chemical surroundings of vacancylike defects. ${ }^{8,9}$ The present knowledge of positronannihilation defect identification in InP defects may be summarized as follows. (i) Vacancy defects are usually present at low concentration in as-grown $p$-type SI materials, and $n$ type materials giving mean lifetimes in the range $243-247$ ps which are longer than the bulk lifetime (236-241 ps), ${ }^{10,11}$ although it appears that such trapping is not present in all material. ${ }^{12,13}$ The typical positron lifetime associated with the trapping sites is in the range 265-275 ps, suggestive of either $V_{\mathrm{In}}$ or $V_{\mathrm{P}}$ monovacancy trapping. (ii) The mean lifetimes found in $n$-type and SI materials are generally a few ps longer than those in $p$-type materials, indicating the presence of more trapping centers. ${ }^{10,11}$ Some $n$-type trapping centers can thus be associated with the phosphorus vacancy $V_{\mathrm{P}}$ [positron lifetime $\sim 267$ ps (Ref. 9)] that is expected to become neutral or negatively charged with the Fermi energy in either the middle or the top of the band gap. ${ }^{14,15}$ The indium vacancy $V_{\text {In }}$ [positron lifetime $\sim 283$ ps (Ref. 9)] appears to be the dominant trapping center irrespective of conductivity type. (iii) Electron-irradiation studies have revealed that although Frenkel pair defects produced in both sublattices have annealed out by $\sim 250 \mathrm{~K}$, fractions of $V_{\text {In }}$ and $V_{\mathrm{P}}$ produced under irradiation are not annealed out at room temperature, and that these are stable to higher temperatures. ${ }^{10,12,16,17}$ This concurs with the observation that both $V_{\text {In }}$ and $V_{\mathrm{P}}$ (or related complexes) are stable, and are found in as-grown material.

In this work, positron-annihilation lifetime spectroscopy has been employed, probably for the first time, to study defect configurations in $n$-type undoped LEC-grown InP that has been made semi-insulating through long-time annealing at $950{ }^{\circ} \mathrm{C}$. As with other observers, ${ }^{10,11}$ we find in the asgrown state a degree of positron trapping into what are probably either $V_{\text {In }}$ or hydrogen- $V_{\text {In }}$ complexes. The infrared measurements reveal the presence of the shallow donor complex $V_{\mathrm{In}} \mathrm{H}_{4}$ that is dominant in making the as-grown material $n$ type. ${ }^{18,19}$ The annealed material reveals both a significant increase in the mean positron lifetime, indicating increased vacancy trapping, and a total absence of the $V_{\mathrm{In}} \mathrm{H}_{4}$ complex. 
These results are found to be in good agreement with the recently proposed mechanism of $\mathrm{P}_{\mathrm{In}}$ antisite formation, which shows that the $V_{\mathrm{In}} \mathrm{H}_{4}$ complex dissociates into $V_{\text {In }} \mathrm{H}_{n}^{(3-n)-}(0 \leqslant n \leqslant 3)$ deep acceptorlike defects upon annealing. The midgap $\mathrm{P}_{\mathrm{In}}$ antisite deep donor forms through the recombination of a $V_{\mathrm{In}}^{3-}$ with a neighboring $\mathrm{P}$ atom to such a degree that the remaining annealed-in deep acceptors are compensated resulting in the semi-insulating form. ${ }^{19}$ The positron lifetime results also indicate a longer 330-ps component that could possibly be associated with the $V_{\mathrm{In}} V_{\mathrm{P}}$ divacancies, and the removal of $\mathrm{P}$ atoms from their correct lattice sites.

\section{EXPERIMENT}

The sample used in this work was $n$-type $(n=3.4$ $\times 10^{15} \mathrm{~cm}^{-3}$ ) undoped LEC-grown InP, grown by the phosphorus in situ injection method. ${ }^{20}$ A Bio-Rad Hall system was used to measure the resistivity and carrier mobility which were found to be $0.5 \Omega \mathrm{cm}$ and $3740 \mathrm{~cm}^{2} \mathrm{~V}^{-1} \mathrm{~s}^{-1}$, respectively. The sample was annealed by placing it into a quartz tube along with a quantity of red phosphorus. The samples were then annealed at $950^{\circ} \mathrm{C}$ for $95 \mathrm{~h}$ at which the phosphorus ambient was at a pressure of 60 mbar and then cooled down to room temperature at the rate of $35 \mathrm{~K} \mathrm{~s}^{-1}$. Infrared Fourier transmission measurements were carried out before and after annealing under vacuum using a NIC-170 spectrometer, with the sample at $20 \mathrm{~K} .{ }^{19}$

Positron-lifetime measurements were carried out using a fast-fast lifetime spectrometer with a resolution (full width at half maximum) of $235 \mathrm{ps}$. A $3-\mu \mathrm{Ci}{ }^{22} \mathrm{NaCl}$ positron source was directly deposited onto the surface of the sample in order to avoid source annihilations and the need for any correction. The samples were mounted on the copper cold finger of a closed-cycle He refrigerator cryostat for low temperature measurements. Each lifetime spectrum contained $2 \times 10^{6}$ counts and was analyzed using the program of POSITRONFIT. ${ }^{21}$

\section{RESULTS AND DISCUSSION}

\section{A. As-grown material}

In a defect-free crystal, positrons are delocalized and annihilate, with a single lifetime component. When there are defect-trapping centers present, where the electron density is lower, positrons will be trapped into localized states with decreased annihilation rates. The average lifetime therefore increases, and the lifetime spectrum can in general be decomposed into several exponential components. The average lifetime, being the mass center of the spectrum,

$$
\tau_{\mathrm{av}}=\sum_{i} N_{i} t_{i} / \sum_{i} N_{i}
$$

can be obtained directly from a measured spectrum. $N_{i}$ is the number of events at the $i$ th time interval $t_{i}$ from some suitable post-time-zero channel. This makes $\tau_{\text {av }}$ a quantity of high statistical accuracy and one independent of the decomposition process. ${ }^{8}$ Figure 1(a) shows an average lifetime of 245 ps that varies little over the entire temperature range.

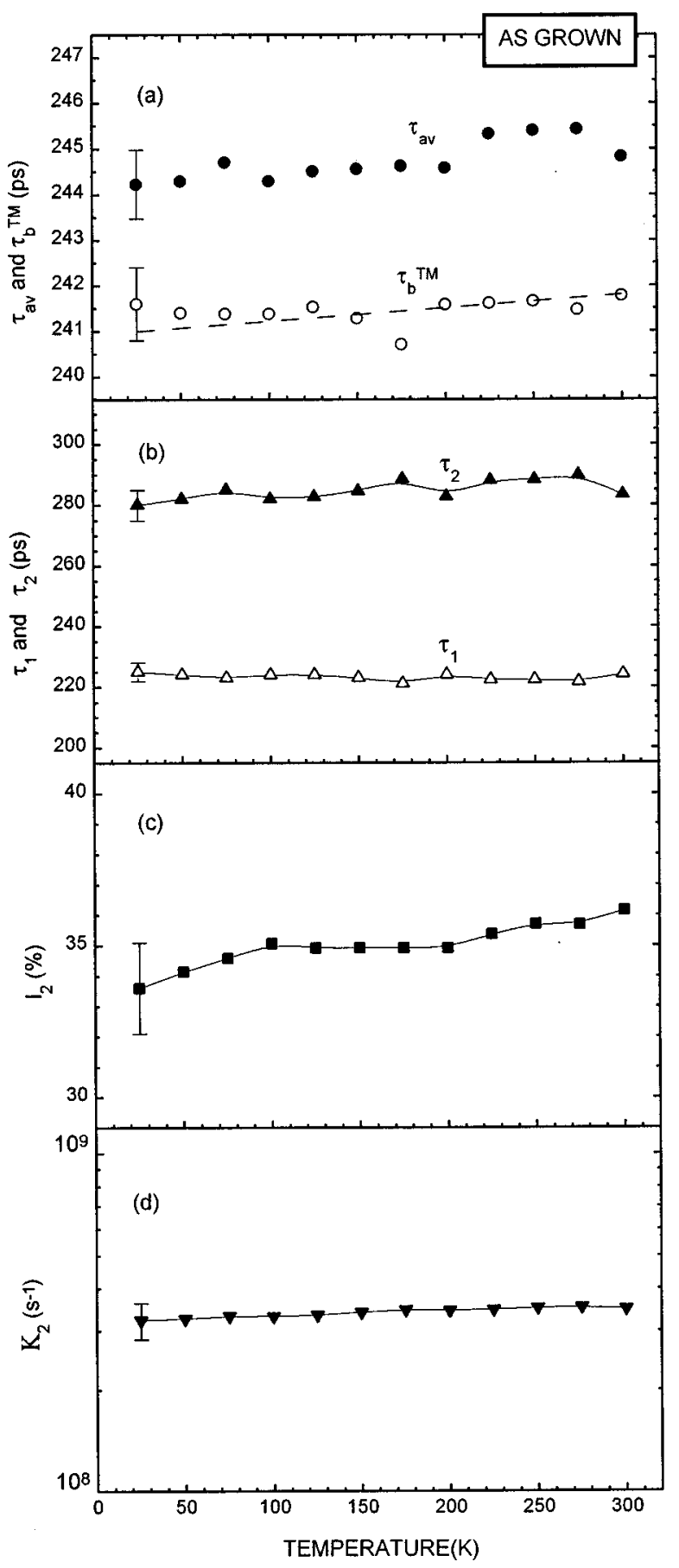

FIG. 1. Positron-lifetime results as a function of measurement temperature for the as-grown state of $n$-type LEC InP. (a) The ideal (no-trapping) positron bulk lifetime $\tau_{b}^{\mathrm{TM}}$ calculated from the twostate trapping model, and the positron average lifetime $\tau_{\mathrm{av}}$. (b) Defect lifetime $\tau_{2}$ and bulk state lifetime $\tau_{1}$ as obtained from two exponential component fit. (c) Relative intensity of the defect component $I_{2}$. (d) The defect trapping rate based on the two-state trapping model.

It was found that before the sample was annealed the lifetime spectra, measured in the temperature range of 25$300 \mathrm{~K}$, could be fitted well by a sum of two exponential components. The mean lifetimes $\tau_{1}$ and $\tau_{2}$ thus obtained are shown in Fig. 1(b), together with the intensity of the longer component $I_{2}$ in Fig. 1(c). It is reasonable to assume the 
simple two-state trapping model (TM) in which positrons are transferred at some rate $\kappa$ from the bulk state to the defect state. ${ }^{8}$ According to this model the bulk lifetime of positron in a nondefected crystal is given by:

$$
\tau_{b}^{\mathrm{TM}}=\left(I_{1} / \tau_{1}+I_{2} / \tau_{2}\right)^{-1},
$$

which is also shown in Fig. 1(a). The value of $\tau_{b}^{\mathrm{TM}}$ is seen to be $\sim 241$ ps across the entire temperature range, with a slight shortening at lower temperatures consistent with lattice contraction. The important point is that the average lifetime is 3-4 ps longer than the delocalized bulk state lifetime as a result of positron trapping by defects. The value of 241 \pm 1 ps for $\tau_{b}^{\mathrm{TM}}$ is in reasonable agreement with the theoretical value $240 \mathrm{ps},{ }^{9}$ and estimates from other studies on asgrown material that suggest a value in the range 234-241 ps. ${ }^{10-12,16}$

As seen from Fig. 1(b), spectral decomposition reveals a positron lifetime of $282 \pm 5 \mathrm{ps}$, with an intensity of around $35 \%$. Defect lifetimes around this value have often been reported in electron-irradiated material, and have been associated with the indium vacancy $V_{\text {In }}$. For example, Törnqvist et al. attributed a 283 7 -ps component to $V_{\text {In }},{ }^{17}$ while Bretagnon, Dannefaer, and Kerr attributed second lifetime components in the range 263-272 ps to $V_{\text {In }}$ (Ref. 10) and $V_{\text {In }}$ interstitial complexes. ${ }^{16}$ Chen, Hu, and Wang, finding a similar $\sim 270$-ps component in as-grown InP of all conductivity types, concluded that the defect center must be dominantly due to $V_{\mathrm{In}}$ since $V_{\mathrm{P}}$ would be positively charged and repulsive to positrons in $p$-type material. ${ }^{11}$ The $V_{\text {In }}$ assignment is also supported by theoretical calculations that place the lifetime of positrons trapped in $V_{\text {In }}$ at $280-290$ ps. ${ }^{19,22}$ The evidence is thus compelling that the trapping site we observe in the as-grown state is, even if not the isolated $V_{\text {In }}$ some complex involving $V_{\text {In }}$.

To estimate the concentration of the $V_{\text {In }}$-related trapping site, we first compute the trapping rate. This may be done using the formula

$$
\kappa(T)=\frac{\tau_{\mathrm{av}}-\tau_{b}^{\mathrm{TM}}}{\tau_{2}-\tau_{\mathrm{av}}} \frac{1}{\tau_{b}^{\mathrm{TM}}},
$$

which is derived from the simple two-state trapping model. ${ }^{8}$ The variation of $\kappa$ with $T$ is shown in Fig. 1(d), and is seen to change little over the entire temperature range. The concentration of positron trapping defects $C$ can be obtained by

$$
\kappa=\left(\mu_{v} / N_{\mathrm{at}}\right) C,
$$

where $\kappa$ is the defect trapping rate, $\mu_{v}$ is the specific positron trapping rate, and $N_{\text {at }}$ is the number of sublattice atoms in unit volume. ${ }^{23}$ Taking $\mu_{v}$ to be $4.5 \times 10^{14} \mathrm{~s}^{-1}$ for neutral vacancies, ${ }^{24}$ and $N_{\text {at }}$ to be $1.98 \times 10^{22} \mathrm{~cm}^{-3}$ for InP, the concentration of positron vacancy traps is found to be $\sim 1.5$ $\times 10^{16} \mathrm{~cm}^{-3}$.

A complication with assigning the observed 282-ps component to $V_{\text {In }}$ is that the expected charge state of this defect, as judged from theoretical ionization levels, ${ }^{15}$ is $(3-)$, and as such a strong increase in the positron trapping related parameter $I_{2}$ should be expected at low temperature. ${ }^{23}$ Since our experimental results reveal no such variation of $I_{2}$, and indeed a slight decrease at low temperature is noted, the

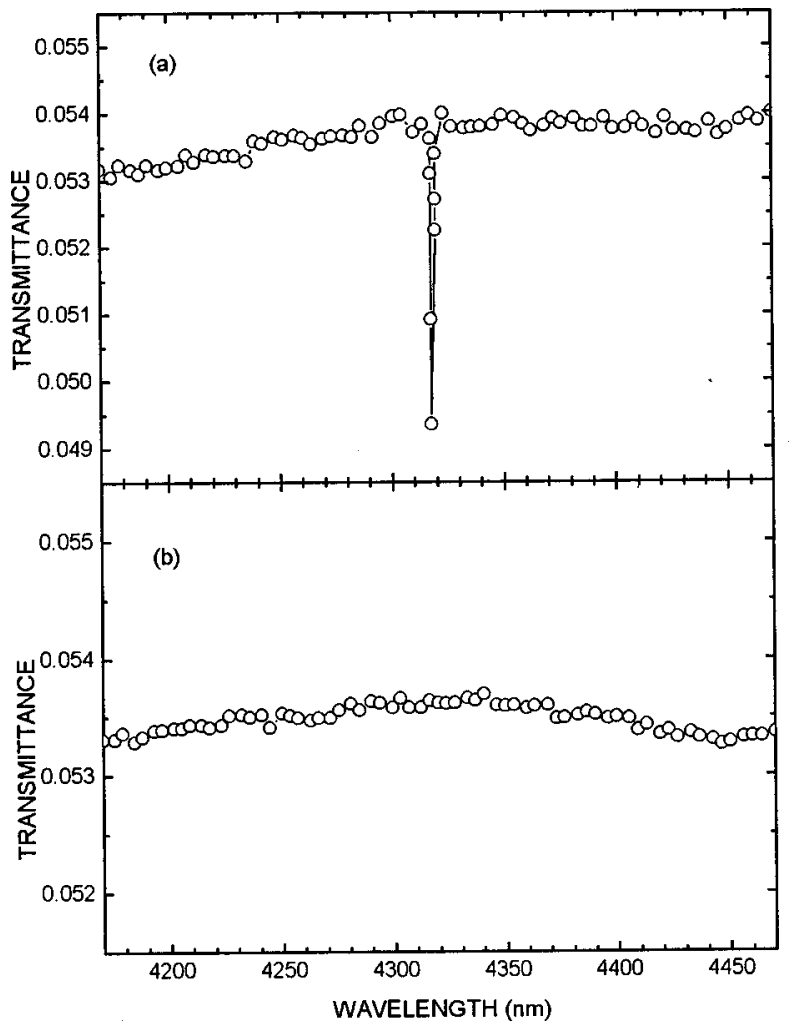

FIG. 2. FT-IR transmission spectra taken (a) before annealing and (b) after annealing. The absorption peak seen in (a) at the wavelength $4320 \mathrm{~nm}$ is the local vibration mode of $V_{\text {In }} \mathrm{H}_{4}$ complex.

suggestion of $V_{\operatorname{In}}^{3-}$ trapping is questionable. However, a number of observations can be made. The first is that impurity shallow acceptors (mainly $\mathrm{Zn}, \mathrm{Mg}, \mathrm{Ag}$, and $\mathrm{Ca}$ ) are known to be present in the InP material ${ }^{19}$ at the 2 $\times 10^{16}-\mathrm{cm}^{-3}$ level. These, being $(1-)$ in their ionized states, are expected to form shallow positron traps and thus compete with vacancy trapping as the sample temperature is lowered, thus removing the expected temperature dependence. Second, the $V_{\text {In }}$ defect may not be in its simple isolated form, but is likely to be complexed with hydrogen which is abundant in as-grown material. The presence of hydrogen atom(s) in association with $V_{\text {In }}$ may only cause a small difference from the lifetime value. One form of $V_{\text {In }}$ complex, $V_{\mathrm{In}} \mathrm{H}_{3}$, is neutrally charged ${ }^{18}$ and could be the sought for trapping site. The other species $V_{\text {In }} \mathrm{H}_{2}$ and $V_{\text {In }} \mathrm{H}$ are expected to be singly and doubly negatively charged, respectively, and their presence would imply the need to invoke shallow trapping once again to explain the observed $I_{2}$ temperature dependence.

The presence of hydrogen in the as-grown InP in the form of the $V_{\mathrm{In}} \mathrm{H}_{4}$ complex is shown clearly in Fig. 2(a) through its Fourier transform (FT)-IR absorption peak at wavelength $4320 \mathrm{~nm}$. From the estimated absorption coefficient of this line, the intensity of this peak gives a $V_{\text {In }} \mathrm{H}_{4}$ concentration of at least $\sim 10^{16} \mathrm{~cm}^{-3}$. It is tempting to consider this complex as the positron trapping center, especially because its concentration is so close to that estimated from the positron trapping rate. However, there is a major objection to such an assignment. In the first place it is known that $V_{\text {In }} \mathrm{H}_{4}$ is dominantly ionized at room temperature, and is a major contribu- 
tor to the materials' $n$-type conductivity. ${ }^{19}$ It is thus in the positively charged state $\left(V_{\mathrm{In}} \mathrm{H}_{4}\right)^{+}$state, ${ }^{18}$ and as such is not expected to trap positrons. The fraction of the complex in the $\left(V_{\text {In }} \mathrm{H}_{4}\right)^{0}$ state is estimated to be less than $10 \%$, and is too small to explain the observed concentration of trapping centers.

\section{B. Annealed semi-insulating material}

After the sample was annealed, the material became semiinsulating with a room-temperature resistivity of 2.8 $\times 10^{7} \Omega \mathrm{cm}$. At the same time, as shown in Fig. 2(b), the FT-IR measurements gave no evidence of any $V_{\text {In }} \mathrm{H}_{4}$ absorption peak, indicating a thermal dissociation of this complex had taken place. ${ }^{19}$ These changes can be explained by a general compensation mechanism model, which assumes that an annihilation of intrinsic defects occurs upon annealing, and that the trap levels created play important roles in the compensation. ${ }^{25}$ The more specific model of compensation will be discussed below.

The results of the positron-annihilation lifetime measurements, in the temperature range of $25-300 \mathrm{~K}$ after annealing, are shown in Fig. 3. As seen from Fig. 3(a), the average positron lifetime $\tau_{\mathrm{av}}$ has increased from the as-grown value of $245 \mathrm{ps}$ to $250 \mathrm{ps}$, indicating a general increase in the degree of positron defect trapping. It was also found that two exponential components could no longer fit the spectra properly, and spectral decomposition into three components revealed a third component of lifetime $\tau_{3} \cong 330 \mathrm{ps}$, with an intensity of $\sim 5 \%$. The second component remained with a very similar lifetime $\tau_{2} \cong 285$ ps. The 330 -ps component is indicative of a defect with a larger open volume, and suggests a divacancy trapping center. Indeed it has been reported that the divacancy $\left(V_{\mathrm{In}} V_{\mathrm{P}}\right)$ and divacancy-interstitial complexes in InP have positron lifetimes of 338 and 340 ps, respectively. ${ }^{16,22}$ The presence of the 285-ps component suggests that some $V_{\mathrm{In}}$-related defects remain after annealing.

Solution of the positron annihilation rate equations in a three-state trapping model, assuming two different trapping rates $\kappa_{2}$ and $\kappa_{3}$ into the two different sites, yields the set of equations ${ }^{8}$

$$
\begin{gathered}
\tau_{b}^{\mathrm{TM}}=\left(I_{1} / \tau_{1}+I_{2} / \tau_{2}+I_{3} / \tau_{3}\right)^{-1}, \\
\kappa_{2}=\left(1 / \tau_{1}-1 / \tau_{2}\right) I_{2}, \\
\kappa_{3}=\left(1 / \tau_{1}-1 / \tau_{3}\right) I_{3},
\end{gathered}
$$

where $I_{2}$ and $I_{3}$ are the relative intensities of the two defect components. Figure 3(a) shows that the bulk lifetime $\tau_{b}^{\mathrm{TM}}$ is still around 241 ps. Since the value derived from the threestate trapping model is in good agreement with the preanneal value of $\tau_{b}^{\mathrm{TM}}$, the reasonableness of the three-state model in describing the system after annealing is indicated. The fitting results for both as-grown and annealed cases are listed in Table I. The values of $\kappa_{2}$ and $\kappa_{3}$, calculated in this model, are shown in Fig. 3(d). These values allow us to estimate the concentration of the $V_{\mathrm{In}}$-related defect $(285 \mathrm{ps})$, and of the divacancy (330 ps) in the semi-insulating form. Using the same specific trapping rate as used for the as-grown case, the values obtained are $1.6 \times 10^{16}$ and $3.0 \times 10^{15} \mathrm{~cm}^{-3}$, respectively.

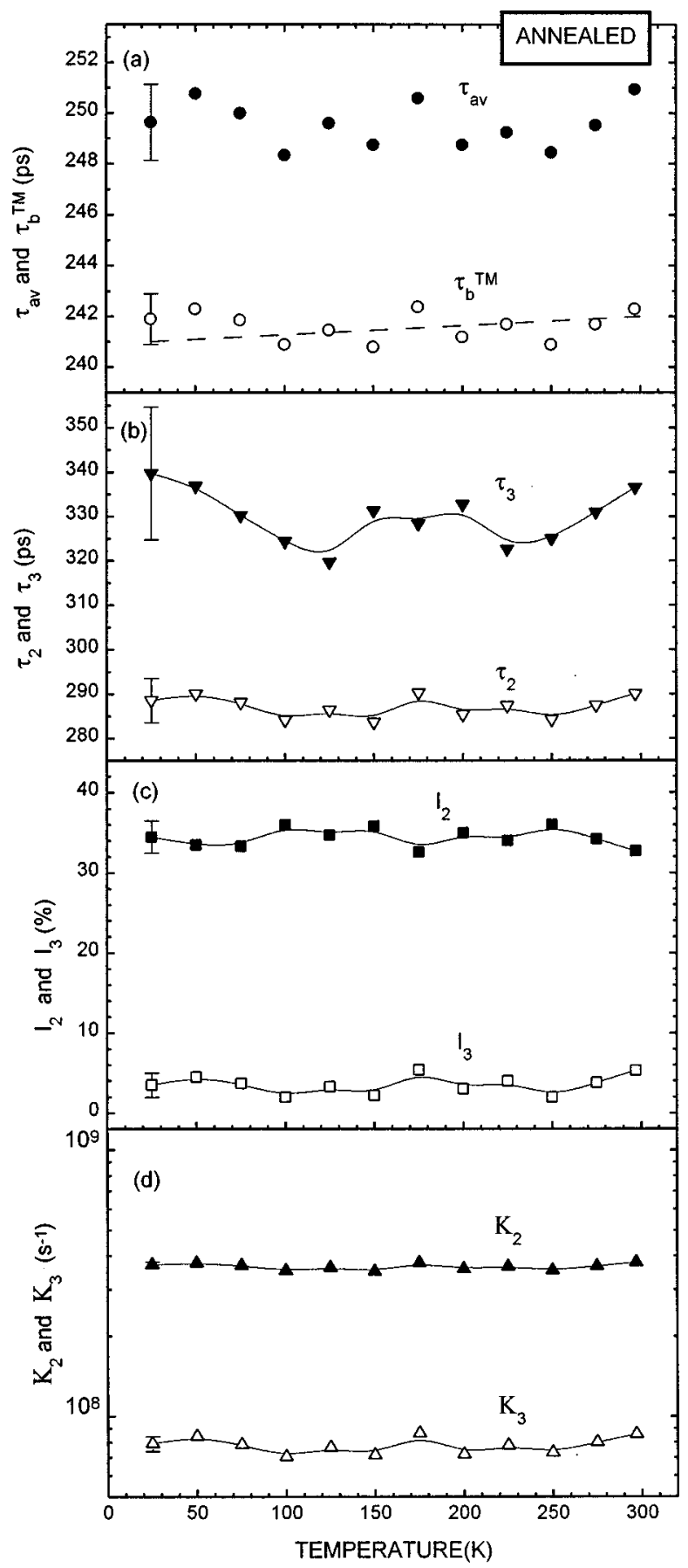

FIG. 3. Positron-lifetime results as a function of measurement temperature for the annealed SI-InP. (a) The ideal (no-trapping) positron bulk lifetime $\tau_{b}^{\mathrm{TM}}$, calculated from the three-state trapping model, and the positron average lifetime $\tau_{\mathrm{av}}$. (b) Defect lifetimes $\tau_{2}$ and $\tau_{3}$ as obtained from the three-exponential-component fit. (c) Relative intensities $I_{2}$ and $I_{3}$ of the two observed defect components. (d) The trapping rates $\kappa_{2}$ and $\kappa_{3}$ of the two defects based on the three-state trapping model.

Following the model of Ref. 19 the disappearance of the $V_{\text {In }} \mathrm{H}_{4}$ FT-IR absorption peak arises from the dissociation of the $V_{\text {In }} \mathrm{H}_{4}$ complexes into $V_{\mathrm{In}} \mathrm{H}_{n}^{(3-n)-}(0 \leqslant n \leqslant 2)$ complexes, and finally the isolated $V_{\mathrm{In}}^{3-}$ during annealing:

$$
\begin{aligned}
4\left(V_{\mathrm{In}} \mathrm{H}_{4}\right)^{+}+10 e^{-} \rightarrow & \left(V_{\mathrm{In}} \mathrm{H}_{3}\right)^{0}+\left(V_{\mathrm{In}} \mathrm{H}_{2}\right)^{-} \\
& +\left(V_{\mathrm{In}} \mathrm{H}\right)^{2-}+V_{\mathrm{In}}^{3-}+10 \mathrm{H} \uparrow .
\end{aligned}
$$


TABLE I. Positron annihilation lifetime results before and after annealing.

\begin{tabular}{lcc}
\hline \hline & Before annealing & After annealing $\left(950^{\circ} \mathrm{C}\right)$ \\
\hline$I_{2}(\%)$ & 35 & 33 \\
$I_{3}(\%)$ & $/$ & 5 \\
$\tau_{1}(\mathrm{ps})$ & 223 & 218 \\
$\tau_{2}(\mathrm{ps})$ & 282 & 285 \\
$\tau_{3}(\mathrm{ps})$ & $/$ & 335 \\
$\kappa_{2}\left(\mathrm{~s}^{-1}\right)$ & $3.3 \times 10^{8}$ & $3.6 \times 10^{8}$ \\
$\kappa_{3}\left(\mathrm{~s}^{-1}\right)$ & $/$ & $7.5 \times 10^{7}$ \\
$\tau_{b}^{\mathrm{TM}}(\mathrm{ps})$ & 241 & 241 \\
$\tau_{\mathrm{av}}(\mathrm{ps})$ & 245 & 250 \\
\hline \hline
\end{tabular}

The $V_{\mathrm{In}}^{3-}$ then either captures an interstitial $\mathrm{P}_{i}$ atom or a neighboring $\mathrm{P}_{\mathrm{P}}$ atom to form the EL2-like antisite defect $\left(\mathrm{P}_{\mathrm{In}}\right)$

$$
\begin{gathered}
V_{\mathrm{In}}^{3-}+\mathrm{P}_{i} \rightarrow \mathrm{P}_{\mathrm{In}}^{2+}+5 e, \\
V_{\mathrm{In}}^{3-}+\mathrm{P}_{\mathrm{P}} \rightarrow \mathrm{P}_{\mathrm{In}}^{2+}+V_{\mathrm{P}}^{+}+6 e .
\end{gathered}
$$

The compensation of hydrogen associated $V_{\text {In }}$ deep acceptor levels and other residual acceptor impurities by the EL2-like $\mathrm{P}_{\text {In }}$ antisite deep donor produces the semi-insulating state.

The above model allows a relatively easy interpretation of the positron lifetime data. The $V_{\mathrm{In}} \mathrm{H}_{n}^{(3-n)-}$ products of the above dissociation, being $V_{\text {In }}$ related, are anticipated to be additional positron traps with lifetimes of around 280-290 ps. However, the trapping rate into this monovacancy component has not increased significantly, which would suggest that the hydrogen evolution pathway expressed in reaction (6) is a secular equilibrium, with the concentration of intermediate species $V_{\text {In }} \mathrm{H}_{n}^{(3-n)-}$ remaining approximately constant. With regard to the 330-ps lifetime component, reaction
(8) gives a possible source of a divacancy defect. $V_{\mathrm{P}}$ formed through this reaction could be in the vicinity of an indium vacancy and form the $V_{\mathrm{In}} V_{\mathrm{P}}$ divacancy or hydrogen associated forms of this defect. Clearly more studies at intermediate annealing times could give further evidence to support this picture.

\section{CONCLUSION}

Positron-lifetime measurements carried out on LECgrown InP have shown that the average positron lifetime increases from 245 to 250 ps upon annealing, manifesting stronger positron trapping effects in the annealed state. Two defect components are separated in the annealed semiinsulating state. The first is of similar structure to the trapping center found in the as-grown InP, and has a lifetime $\sim 285$ ps. It is argued that this is most likely to be due to hydrogen $V_{\text {In }}$ complexes of the form $V_{\mathrm{In}} \mathrm{H}_{n}^{(3-n)-}(0 \leqslant n$ $\leqslant 3$ ), since the lifetime value is close to the positron lifetimes of indium-vacancy-related defects seen by others. A second defect lifetime component of $\sim 330 \mathrm{ps,} \mathrm{found} \mathrm{in}$ semi-insulating form, is most likely to be due to the $V_{\text {In }} V_{\mathrm{P}}$ divacancy or possibly related complexes. Both these defects are suggested to originate from the thermal dissociation of the $V_{\mathrm{In}} \mathrm{H}_{4}$ complex. ${ }^{19}$ Positron trapping rates into both defect types are found to be essentially independent of temperature over the wide temperature range of $25-300 \mathrm{~K}$, indicating either that these defects are neutrally charged, or that competing shallow traps exist having a trapping rate which follows that of the vacancy defects.

\section{ACKNOWLEDGMENTS}

The authors wish to acknowledge valuable financial support from the HKU CRCG and the Hong Kong RGC in performing this work.
${ }^{1}$ D. E. Holmes, R. G. Wilson, and P. W. Yu, J. Appl. Phys. 52, 7409 (1981).

${ }^{2}$ G. Marrakchi, K. Cherkaoui, A. Karoui, G. Hirt, and G. Müller, J. Appl. Phys. 79, 6947 (1996).

${ }^{3}$ D. Hofmann, G. Müller, and N. Streckfuss, Appl. Phys. A: Solids Surf. 48, 314 (1989).

${ }^{4}$ G. Hirt, D. Hofmann, F. Mosel, N. Schafer, and G. Müller, J. Electron. Mater. 20, 1065 (1991).

${ }^{5}$ K. Kainosho, H. Shimakura, H. Yamamoto, and O. Oda, Appl. Phys. Lett. 59, 932 (1991).

${ }^{6}$ R. Fornari, A. Brinciotti, E. Gombia, R. Mosca, and A. Sentiri, Mater. Sci. Eng., B 28, 95 (1994).

${ }^{7}$ A. Hruban, St. Strzelecka, W. Wegner, M. Gladysz, W. Orlowski, M. Piesa, and A. Mirowska, in Proceedings of the 8th Conference on Semi-insulating III-V Materials, Warsaw, Poland, 1994, edited by M. Goldlewski (World Scientific, Singapore, 1994), p. 43.

${ }^{8}$ See, for review, P. Hautojärvi and C. Corbel, in Positron Spectroscopy of Solids, Proceedings of the International School of Physics, "Enrico Fermi", edited by A. Dupasquier and A. P. Mills (North-Holland, Amsterdam, 1994), Course CXXV.
${ }^{9}$ M. Alatalo, H. Kauppinen, K. Saarinen, M. J. Puska, J. Mäkinen, P. Hautojärvi, and R. M. Nieminen, Phys. Rev. B 51, 4176 (1995).

${ }^{10}$ T. Bretagnon, S. Dannefaer, and D. Kerr, J. Appl. Phys. 73, 4697 (1993).

${ }^{11}$ Z. Q. Chen, X. W. Hu, and S. J. Wang, Appl. Phys. A: Solids Surf. 66, 435 (1998).

${ }^{12}$ A. Polity and T. Engelbrecht, Phys. Rev. B 55, 10480 (1997).

${ }^{13}$ G. Dlubek and R. Krause, Phys. Status Solidi A 102, 443 (1987).

${ }^{14}$ Z. Q. Chen, X. W. Hu, S. J. Wang, and S. Q. Li, Solid State Commun. 99, 745 (1996).

${ }^{15}$ A. P. Seitsonen, R. Virkkunen, M. J. Puska, and R. M. Nieminen, Phys. Rev. B 49, 5253 (1994).

${ }^{16}$ T. Bretagnon, S. Dannefaer, and D. Kerr, J. Appl. Phys. 81, 3446 (1997).

${ }^{17}$ M. Törnqvist, C. Corbel, L. Liszkay, K. Saarinen, and P. Hautojärvi, Mater. Sci. Eng., B 28, 125 (1994).

${ }^{18}$ C. P. Ewels, S. Orberg, R. Jones, B. Pajot, and P. R. Briddon, Semicond. Sci. Technol. 11, 502 (1996).

${ }^{19}$ Y. W. Zhao, X. L. Xu, M. Gong, S. Fung, C. D. Beling, X. D. 
Chen, N. F. Sun, T. N. Sun, S. L. Liu, G. Y. Yang, X. B. Guo, Y. Z. Sun, L. Wang, Q. Y. Zheng, Z. H. Zhou, and J. Chen, Appl. Phys. Lett. 72, 2126 (1998).

${ }^{20} \mathrm{~S}$. Tong-nien, L. Szu-lin, and K. Shu-tseng, in Proceedings of the 2nd Conference on Semi-insulating III-V Materials, Evian, France, 1982, edited by Sherif Makram-Ebeid and Brian Tuck (Shiva, UK, 1982), p. 61.

${ }^{21}$ P. Kirkegaard, M. Eldrup, O. E. Mogensen, and N. J. Pedersen, Comput. Phys. Commun. 23, 307 (1981).
${ }^{22}$ M. J. Puska, S. Mäkinen, M. Manninen, and R. Nieninen, Phys. Rev. B 39, 7666 (1989).

${ }^{23}$ W. Brandt and R. Paulin, Phys. Rev. B 5, 2340 (1972).

${ }^{24}$ M. J. Puska, C. Corbel, and R. M. Nieminen, Phys. Rev. B 41, 9980 (1990).

${ }^{25}$ K. Kainosho, O. Oda, G. Hirt, and G. Müller, in Physics and Applications of Defects in Advanced Semiconductors, edited by M. O. Manasreh, H. J. von Bardeleben, G. S. Pomerenke, and M. Lannoo, MRS Symposia Proceedings No. 325 (Materials Research Society, Pittsburgh, 1994), p. 101. 